\title{
High Resolution X-ray UltraMicroscopy and Tomographic Reconstruction of Micro-Craters Preserved in Metallic Foils
}

\author{
J. Sheffield-Parker, ${ }^{*}$ G.A. Graham, ${ }^{* *}$ L.A. Brownlow, ${ }^{*}$ and A.T. Kearsley***
}

* XRT Ltd, Port Melbourne, VIC 3207, Australia

** Institute of Geophysics \& Planetary Physics, Lawrence Livermore National Laboratory, PO Box 808, Livermore, CA 94551, USA

*** Electron Microscopy \& Mineral Analysis, Mineralogy Department, The Natural History Museum, London, SW7 5BD, UK

The study of micro-craters generated as a result of impact events has a wide range of applications from forensic engineering [1] to the aerospace industry, and in particular to impact damage to satellites and spacecraft in low-Earth-orbit [2]. Hypervelocity collisions of natural micrometeoroids and artificial orbital debris (e.g. paint fragments) can pose a hazard to the operational capabilities of a satellite or space vehicle [3]. As a result, when space exposed surfaces are successfully retrieved from low-Earth orbit they are subjected to detailed post-flight investigations. As part of these postflight investigations, optical and scanning electron microscopy based techniques are implemented to investigate micrometer to millimeter sized impact craters [4]. These microscopy studies focus on the search for remnant projectile material and the acquisition of crater geometries such as crater diameter $\left(D_{c}\right)$ and crater depth $(p)$. Typically $p$ measurements require prior cross-sectional destructive sample preparation, however here we discuss the application of high resolution x-ray microscopy to study intact micro-craters preserved in aluminum foils [5].

Conventional X-ray imaging is based on the absorption of radiation with image contrast produced as a result of the varying composition, thickness or density of the sample. While this is a well established technique, the method suffers severe limitations when studying low density and weakly absorbing materials which provide little attenuation of the X-ray signal. X-ray phase contrast imaging is a relatively new area of X-ray imaging science which is well suited to the study of such materials. Historically phase contrast imaging was considered to only be achievable using high brightness monochromatic X-ray sources, such as those achievable with a synchrotron, however more recent work has shown that phase contrast imaging can also be achieved using laboratory scale polychromatic X-ray sources [6].

The X-ray ultraMicroscope (XuM) is a high resolution point projection X-ray microscope which uses a scanning electron microscope as a host. The electron beam is focused onto a small target Xray source and the generated X-rays pass though the sample (100 micrometer thick Al 1100 series foil) to form a projected image containing both phase and absorption contrast onto the direct detection CCD X-ray detector (Fig. 1). In addition to through-thickness planar imaging of the impact crater, the geometry of the XuM also lends itself to the acquisition of rotational data sets rotated about the vertical axis. A rotational data set acquired in this way was subjected to postacquisition phase-retrieval followed by tomographic reconstruction using an implementation of the Feldkamp-Davis-Kress cone beam algorithm [7]. The virtual slices generated during this post processing enabled detail investigation of crater geometries including $p$ measurement (Fig. 2a). Finally the virtual slices were reconstructed to produce rendered tomographic surfaces and volume images of the crater for 3D analysis (Fig. 2b-2c). 
The XuM analysis complements the traditional SEM imaging and analysis techniques applied to micro-crater analysis. Significant capabilities are the ability to form X-ray images showing the internal structure and micro-tomography without the need for destructive cross-sectioning.
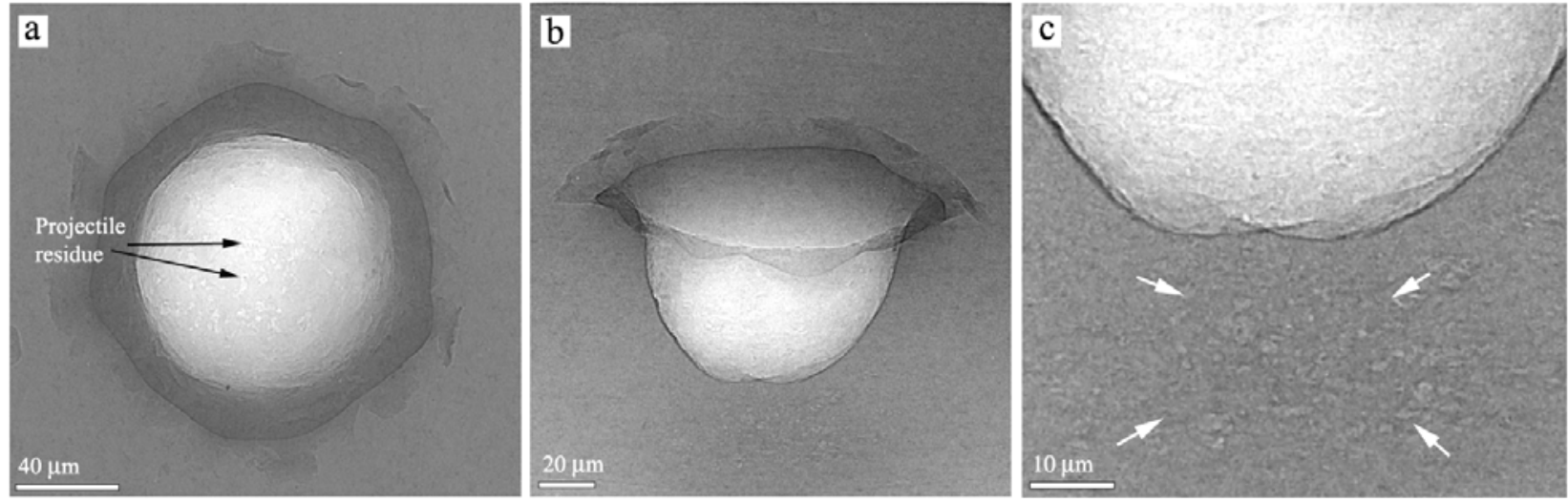

FIG. 1. (a) X-ray image of an impact crater. (b) X-ray image of crater rotated. (c) High magnification X-ray image of the crater base. Microstructure damage to the aluminum substrate can also be observed (indicated by the white arrows).
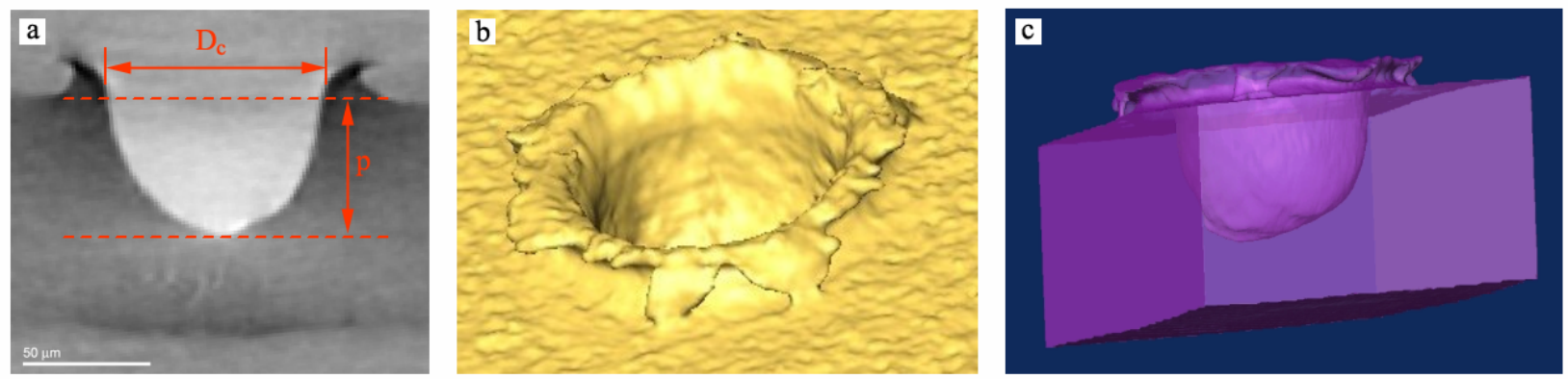

FIG. 2. (a) Reconstructed cross-sectional virtual slice of the impact crater. From this slice accurate measurement of the crater diameter $\left(D_{c}\right)$ and crater depth $(p)$ can be obtained. (b) A tomographic surface reconstruction of the crater. (c) A tomographic volume reconstruction of the crater.

\section{References}

[1] M.T. Baker and J.M. Winn. In Forensic Investigation of Explosions (ed. A. Beveridge), p404442, CRC Press, Florida, 1998.

[2] J.C. Mandeville and L. Berthoud, Advances in Space Research 16 (1995) 67.

[3] R.D. Caswell et al., Advances in Space Research 33 (2004) 1507.

[4] A.T. Kearsley et al., Advances in Space Research 35 (2005) 1254.

[5] G.A. Graham et al., Lunar and Planetary Science XXXVII (2006) abstract \# 2280.

[6] S.W. Wilkins, et al., Science 384 (1996) 335.

[7] L.A. Feldkamo et al., Journal of the Optical Society America 6 (1984) 612.

[8] This work was performed under the auspices of the U.S. Department of Energy, National Nuclear Security Administration by the University of California, Lawrence Livermore National Laboratory under contract No. W-7405-Eng-48. F. Hörz (NASA JSC) is thanked for providing the foil samples used in this study. 